\title{
Entropy
}

ISSN 1099-4300

(C) 2003 by MDPI

www.mdpi.org/entropy

\section{Information and Meaning}

\author{
Christophe Menant
}

Bordeaux France. E-mail: crmenant@free.fr

Received: 10 January 2003 / Accepted: 12 February 2003 / Published: 30 June 2003

\begin{abstract}
We propose here to clarify some of the relations existing between information and meaning by showing how meaningful information can be generated by a system submitted to a constraint. We build up definitions and properties for meaningful information, a meaning generator system and the domain of efficiency of a meaning (to cover cases of meaningful information transmission). Basic notions of information processing are used.
\end{abstract}

Keywords: Meaning, Information, System, Constraint, Generation, Efficiency.

\section{Introduction. Meaning Versus Information}

Information and meanings are important part of the world surrounding us, as well as part of ourselves. It is quite obvious to consider that there are some relations between information and meanings.

Many examples illustrate these relations. When reading a newspaper, we skip items that do not interest us and pay attention to information that has some meaning versus our subjects of interests. These meanings may be different from the ones that have motivated the authors of the articles. Depending if we are on the beach or under shelter, noise from thunderstorm will have different meaning for us. When we talk with other persons, we often want these persons to accept a precise meaning attached to the information we provide. A frog has a visual system able to see object the size of an insect or worm, providing it moves like one. Visual information of food that is not moving has no meaning for a frog. And so on .... On the same token, it is generally agreed upon that information processing machine do not take into account the meaning attached to the information they process. 
Because the meaning related to the information comes from the user of the machine or from the designer. And it cannot be transferred to the machine.

Important work has been done by philosophers and scientists on questions relatives to the meaning of words, sentences or signs. On the side of the philosophers, C. S. Peirce has elaborated at the end of the 19 th century a theory of sign with categories involving meaning and representation [1]. During the 20th century, several theories of meaning have been developed. They recognize that meaning does depend on us humans [2]. Regarding the activity of scientists, Claude E. Shannon, while establishing the mathematical foundations of communication theory, has elaborated in the 1940's a way to measure the content of information in a message. But the meaning of the information was not taken into account as irrelevant to the engineering problem. A few years after, Donald MacKay proposed to look to the meaning of information as related to a target oriented communication [3]. More recently, A. Sharov has analyzed the problem of the sense of information with the help of Biosemiotics [4]. A lot also has been written about meaningful information as feeding our motivation and our beliefs.

But not much has been done regarding the notion of meaning by itself, whatever the information associated to it or the system managing it. It is this aspect of relations linking information and meaning we are interested in. We propose that there can be some common ground for most types of meanings, and that this common ground can be explicited by defining a basic system managing the information and the associated meaning.

\section{The Nature of Meaning. Meaningful Information}

The field covered by the notion of meaning is immense, as meaning can be related to any type of information. Information carried by physical or chemical signals (noise, light, odour, presence of an object), or nervous/hormonal information regulating the functioning of a living organism, or also psychic information populating our mental life (thoughts, ideas, reasoning, fears,...).

The word "meaning" is used in many different ways. Let's begin by analyzing what is generally put under this word. When looking at dictionaries, it appears that the word "meaning" is heavily centered on human. The point is that definitions in dictionaries link the word "meaning" to a performance of human mind. In "Le Vocabulaire technique et critique de la philosophie" (A. Lalande), one can find for the word "meaning": "Function of signs. What a sign represents, sense of a word, of a sentence, etc." The same dictionary gives for the word "sense": "what "means to say", what communicates to the mind a word, a sentence or any other sign playing a similar role".

So, in its common acceptance, we find again that the notion of meaning is linked to information as processed by human. And this link to human introduces well the complexity of the subject of meaning. We all know the difficulties we encounter about the simple fact of trying to correctly understand each other when we communicate. When information is exchanged between two persons, several questions can arise about the meaning related to this information. Should it be considered that the meaning is defined by the person producing the information or by the one receiving it, or by both? or by the context in which the information has been produced ? or even by the history of the subject addressed in the information? Is the content of the meaning explicit or implicit? should we consider several 
levels of meaning, and which ones ? relative to what and for whom ? how can be formalized a possible difference of meaning that will be acceptable for the transmitter of the information as well as for the receiver? Etc. These few questions indicate that the analysis of the notion of meaning is complex, and this up to the point where it becomes rapidly unworkable.

In order to introduce some possible simplification in this area, we feel it is important to consider that the linkage of the notion of meaning to the performances of human attaches implicitly the understanding of meaning to the understanding of human mind.

In fact, most information processed by human implies the existence of mind (intelligence, reason, emotions, thoughts, common sense, ...). So the notion of meaning is linked to the characteristics and performances of human mind. And this linkage, even if it is implicit, has important consequences on the analysis of the notion of meaning. This for the simple reason that we make the notion of meaning imprecise and indefinite by associating it to something we do not clearly understand. Because we do not really know what human mind is.

Human mind is something which is really mysterious. At current level of the development of science, the nature of mind is still to be discovered. Studies on mind are however numerous. Their main characteristic being their diversity (philosophy, neurology, artificial intelligence, psychology, science of knowledge/cognisciences, ...). The results achieved so far by these many fields of research are alas far from delivering an acceptable understanding of the nature of mind. The nature of mind is currently out of the field of scientific knowledge. Consequently, we can say that the current linking of the notion of meaning to the nature of human imposes to the notion of meaning a lot of elements that are not today in the field of scientific knowledge.

So one of the reasons making difficult the study of the notion of meaning is that we closely relate it to the domain of human mind which is unknown. If we want to proceed ahead with the hope of reaching some understanding of "meaning" on a general basis, we must look at the possibility of modelizing it in a domain less complex and better known than the domain of human.

In this context, we feel that the domain of non human living elements is interesting as a starting point for the study of the notion of meaning. And this because the nature of life is better understood by science than is the nature of human. Biology is a science whose base ground is relatively firm. Psychology does not benefit of such firm bases, as lacking an understanding of the nature of human mind. An analysis of the notion of meaning in the framework of non human life will be rooted in a better known domain.

This is what we are going to do in this paper: disconnect the notion of meaning from human and come down the ladder of complexity by linking the notion of meaning to information as processed by non human living elements. But disconnecting "meaning" from human at this stage should not be looked at as putting "meaning" apart from human in our approach. Such a disconnection is temporary. It is done because meaning in human is too complex to be taken as a base on which a modelization of meaning generation can be built directly. Once this modelization established using simpler grounds, we will have to work (beyond this paper) on applying it to human. 
To implement our approach, we must choose a living creature that is far enough from human in terms of performances. In order to satisfy this criteria, we will choose a unicellular animal, a paramecium. A paramecium is a unicellular animal that is able to move in water. The simplicity of this organism makes us sure of its limited performances. Paramecium has no mind to which could be communicated "a word, a sentence or any other sign playing a similar role". The characteristics of the paramecium we are going to take into account are the ones shared with all living organisms: satisfy its vital constraints. Which means the ability to subsist as an individual in the surroundings, and subsist as a species (participate to the reproduction of the species members).

Many experiences have been implemented with paramecia. For instance, it has been shown experimentally that a drop of acid in the water at the vicinity of a paramecium will make it move away, looking for a location in water where there is less acid.

This reaction of the paramecium is understood by noticing that acid water is a hostile environment for paramecia. This reaction of a paramecium moving away from a hostile environment allows us to introduce the notion of meaning for a non human living entity. The acid environment represents for the paramecium an information that will participate to some generation of meaning within the paramecium. A meaning that "has sense of", that "wants to say": "the environment is becoming hostile versus the satisfaction of vital constraints". And this meaning is going to trigger within the paramecium an action aimed at putting it at distance from the acid environment. It is clear that the paramecium does not possess an information processing system that allows it to have access to an inner language. But it is true also that a paramecium has usage of sensors that can participate to some measurement of the environment acidity. The information made available with the help of these sensors will be part of the process that will generate the move of the paramecium in the direction of less acid water.

So we can say that the paramecium has created a meaning related to the acidity of its environment, in connection with the satisfaction of its vital constraints. This example of a paramecium reacting to an environment that impacts the satisfaction of its vital constraints illustrates what we want to consider as being a meaning associated to an information. More precisely, this example brings up several characteristics relative to the notion of meaning we are trying to conceptualize. Let's formulate these characteristics in order to bring up a "systemic aspect", more general than the living element that we have taken as example. These characteristics are five in number and are explicited hereunder (the characteristics relative to the living element taken as example are in parenthesis).

1) A meaning (the environment is becoming hostile versus the satisfaction of vital constraints) is associated with an information (level of acidity in water) which is incident on a system capable of processing the information (the paramecium).

2) Meaning is generated because the information processing system possesses a constraint linked to its nature (vital constraints that are to be satisfied in order to maintain a living nature).

3) A meaning is generated because the incident information has a connection with the constraint of the system (too much acid in the water impacts the satisfaction of the vital constraints of the paramecium). 
4) A meaning is a meaningful information relatively to the constraint of the system (information meaning that the environment becomes hostile versus the satisfaction of the vital constraints).

5) The meaningful information is going to participate to the determination of an action that the system is to implement (move towards a less acid location) in order to satisfy it's constraint (satisfy it's vital constraint).

These five characteristics bring us to propose a definition of meaning in the framework of a relation between an information processing system submitted to a constraint, and an information received by this system.

A meaning is a meaningful information that is created by a system submitted to a constraint when it receives an incident information that has a connection with the constraint. The meaning is formed of the connection existing between the received information and the constraint of the system. The function of the meaningful information is to participate to the determination of an action that will be implemented in order to satisfy the constraint of the system.

Figure 1 illustrates this definition:

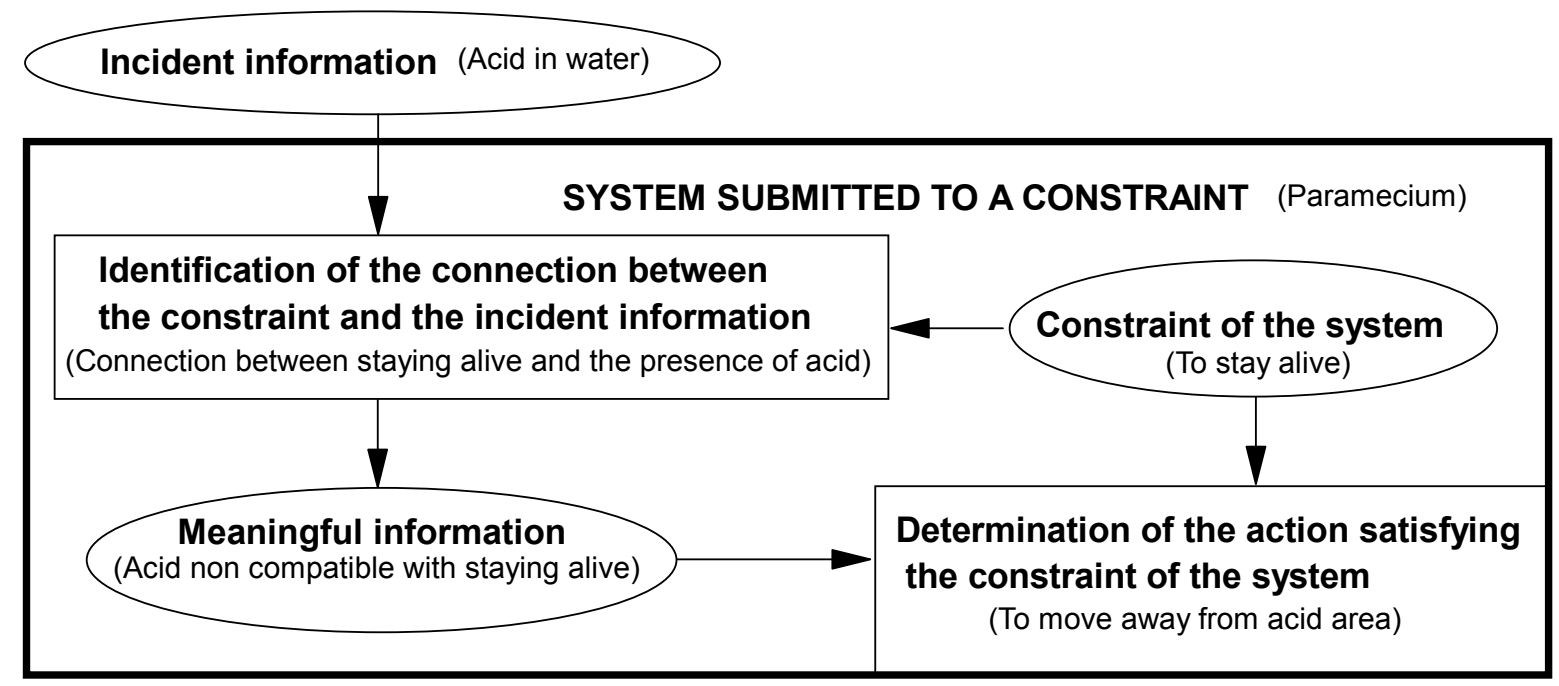

Figure 1. Creation of a meaningful information.

In the following text, we will use indifferently the expressions "meaning" or "meaningful information".

The above definition of meaning calls for the following precision and complements:

- The meaningful information is created by the system in the purpose of having it's constraint satisfied. It is the existence of the constraint to be satisfied that is the cause of the creation of the meaningful information.

- The meaningful information is the information generated by the system submitted to a constraint. It is not the incident information, which is only a contributor to the meaningful information generation.

- Information that has not been produced by a system submitted to a constraint is meaningless information. 
- The content of the meaningful information is the connection existing between the received information and the constraint of the system. If there is no connection between the received information and the constraint, there is no creation of meaningful information.

- A given system can present several connections between its constraint and a received information. The system will then generate several meanings as answer to the incident information.

- A meaningful information is meaningful relatively to the constraint of a system, and only relatively to this constraint. Calling $\mathrm{S}$ the constraint of the system, we will write "meaningful (S) information" or "meaning (S)" the meaning relative to the constraint S. (if several connections exist between the constraint and the received information, we can list the meanings: S1, S2, .. ).

- Some systems can have more than one constraint and/or receive several incident information at the same time. The analysis of meaningful information generation need to be done for each couple (received information, constraint).

- The participation of the meaningful information to the determination of the action can be indirect. Several operations can be combined or chained before the formal action in relation with the satisfaction of the constraint is implemented.

- The action that will be implemented to satisfy the constraint can be internal to the system or be external and involve other systems. (In our example with the paramecium, the action is internal as the paramecium is going to move some elements of its body to displace itself).

- An external action can take place on an element outside the system (the paramecium can displace a small element obstructing its movement), or take place via another system in connection with the first system (see paragraph on meaningful information transmission).

- To have identity of meanings, there need to be identity of received information, identity of constraints and identity of connections between received information and constraints.

- The system creating the meaningful information can be part of a bigger system containing other functions.

On the same token, the relations between signal and information need to be explicited. Information can be memorized or transmitted, but information is always carried by a signal. A text in a closed book is memorized information (the printed characters). The text we are reading from a book is transmitted information (the photons reaching our eyes). In both cases, information is carried by a signal. We call signal any variation of energy (ex: sound vibration - noise, voice-, electromagnetic field change light, radio, photons -, chemical diffusion - odor -, presence of an element - ink, protein -...). The origin of the signal is the transmitter, which is the source of the energy variation. By associating an information to a signal, we define information as the content of the energy variations of the signal (ex: amplitude and modulation of a vibration, variation of chemical concentration at a given point, ink density, molecules in the protein,...). This definition of information applies to all the components of the signal variation. Consequences are that a given signal can carry several different information, and that a signal always carries an information. The elementary information being the signal itself.

It is to be noted here that a signal is not a priori permanent. The variation of energy that has produced the signal can have a limited duration of existence (thunder produced during a thunderstorm 
has a limited duration). But the signal will propagate, and so will continue to exist, even if the energy variation that has produced it does not exist any more (Three $\mathrm{km}$ away, the noise of thunder can be heard 10 seconds after its creation, which is terminated). By the same way, a meaningful information that has been produced by a system will continue to exist even if the system that has originated it does not exist any more. The meaningful information exists as a component of the signal carrying the information. More specifically, a meaningful information as we have defined it will still be meaningful in the absence of the constraint of the system that has produced it. In other words, the meaningful information exists with the signal that carries it, and this even if the system that has created the meaningful information disappears. A meaning (S) stays meaningful in the absence of S.

These precisions regarding the connections between signal and information also show that several information can be carried by the same signal or by different signals (a singer and the orchestra accompanying him can figure on the same recording or on different ones). This remark applies to the incident information and to the meaningful information that exist relatively to the constraint of the information processing system. The meaningful information can be carried by the same signal that the one carrying the incident information, or be carried by another signal. Relatively to the example of the paramecium, the incident information (level of acid in the water) is carried by the signal that is the acid water, but the meaningful information (too much acid) is carried by a biological signal internal to the paramecium. In some other cases, the meaningful information will be carried by the signal carrying the incident information.

In this paper we will deal with the couple formed by the signal and the information carried by the signal. When reading the word "information", one should understand "information carried by the signal".

\section{Meaning Generator System}

The elements introduced here above lead us to introduce more formally a meaningful information generator system, also called a "Meaning Generator System" (MGS) as made of:

- A system submitted to a constraint and able to receive an incident information.

- An information incident on the system.

- An information processing element, internal to the system and capable of identifying a connection between the received incident information and the constraint.

A MGS is represented in Figure 2 where a system submitted to a constraint $\mathrm{S}$ generates a meaningful (S) information. 


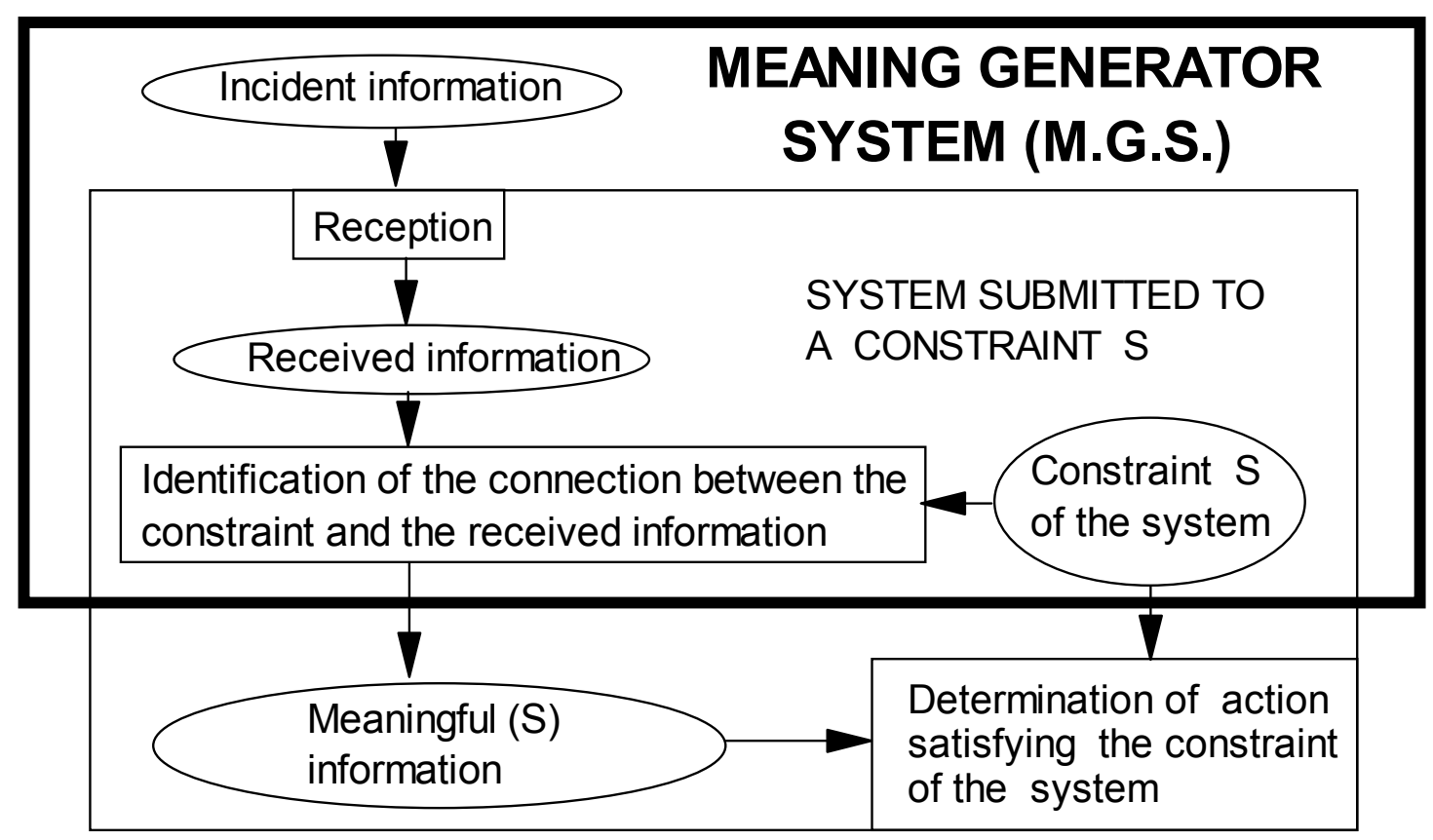

Figure 2. Meaning generator system.

The definition of meaning proposed here above has been built up with an example coming from the non human living world that has been generalized and formalized into a system. This approach is distinct from the Peircean theory of sign (although some elements may be looked at as functionally close):

- Peirce's theory is a theory of sign, and the present paper is centered on meaning. With Peirce, when meaning is explicitly taken into account, it is relatively to the Interpretant. In the present paper, meaning is presented explicitly as a meaningful information. Meaning is defined as the result of a precise information processing done by a system submitted to a constraint.

- The Peircean presentations of sign are done relatively to human (the Interpretant is a human mind, with some indication towards non human minds). As based on mankind, the Peircean theory is rich, powerful and complex. Our introduction of meaning in the present paper is done relatively to an information processing system, with no reference to human for purpose of simplicity regarding the nature of the information processing system. Such a starting point is to be progressively widened by analyzing the different constraints that are to be associated with material systems, living systems and human ones.

- The element of this paper that could be related with the Peircean theory of sign is the Meaning Generator System. This MGS can be compared to a simplified version of the Peircean Interpretant.

The MGS introduced here is simple because its function is limited to generate a meaningful information. It does not include any other function. Other functions like representation, simulation, memory, optimization, action determination, ... belong to other systems that can have close relations with the MGS, but are no part of the MGS. The MGS is simple as compared to many practical cases 
where meaning generation and other functions are often considered together, and where several constraints or received information can interact at the same time with implication of other functions.

We consider that the proposed MGS is usable for all types of meaning generation, assuming we identify and understand the corresponding constraints (which is not currently the case for human where constraints are not clearly understood. See continuation).

\section{Meaning Transmission. Domain of Efficiency of a Meaning}

We have seen above that the meaningful information's role is to participate to the determination of the action that will be implemented in order to satisfy the constraint of the system. It has also been said that this action can take place outside the system, and eventually implicate other systems. This last case corresponds to the transmission of a meaningful information to other systems: the formal action that will satisfy the constraint of the system can come after the transmission of the meaningful information and its reception by other systems. These other systems can also be submitted to specific constraints. In order to take these cases into account, we need to introduce some new notions that will characterize the possibility for a transmitted meaningful information to participate to the determination of an action within another system.

The animal world offers many examples of transmission of meaningful information. An example is the specific sound produced by a male cicada calling female cicadas. This meaningful information is transmitted by the male cicada to the female cicadas in order to have them come for copulation and reproduction in order to satisfy the vital constraint of the species. Another example is given by a bee flying in precise patterns in order to inform the other members of the hive about the location of a source of pollen. This bee dance is a meaningful information that will bring the bees to fly to the source of food to feed the hive, and so contribute to the satisfaction of the vital constraint of the species. Also, pheromones left on the ground by an ant is a meaningful information for the other ants to access food supply (overall indication of best path - the most used -). This information is meaningful versus the survival constraint of the anthill.

But beyond these simple examples is the question concerning the meaning attached to the transmitted information. Will this meaning always be taken into account by the receiver in order to satisfy the constraints of the transmitter? And what if the receiver happens to be submitted to its own constraint, different from the transmitter one? Under which conditions will the receiver be in a position to satisfy the constraint of the transmitter?

These questions are to be looked at in order to address the cases of transmission and reception of meaningful information. To take into account such a subject, let's begin by reminding that a meaning is an information. It is a meaningful information that has been created by a system submitted to a constraint. It has been stated above that a meaningful information keeps its meaningful characteristics even if the system that has generated it disappears. In other words, a meaningful information remains meaningful (S) in a location where the constraint $S$ does not exist. But it is obvious that no action aimed at the satisfaction of the constraint $S$ can take place in a location where the constraint $S$ does not exist (the paramecium cannot try to move from a place where it is not). In other words, this means that 
the meaning $(\mathrm{S})$ will be able to participate to the determination of an action only in the locations where the constraint $S$ exist. In order to clarify these points, we need to introduce the notion of "efficiency of a meaning" that characterizes the possibility for the meaningful information to participate to the determination of an action aimed at satisfying the constraint of the system. We define the efficiency of a meaning as being the aptitude of the meaningful information to participate to the determination of an action aimed at the satisfaction of the constraint of the system. We will note "efficiency (S)" the efficiency of a meaning relatively to the constraint $S$ of the system.

On the same token, we define the "domain of efficiency of a meaning" as being the domain where the meaningful information is capable to participate to the determination of an action aimed at satisfying the constraint of the system. We will note "domain of efficiency (S)" the domain of efficiency of a meaning relatively to the constraint $S$ of a system. And we will state that the domain of efficiency (S) is the location where the constraint $S$ of the system is existing. In other words, the meaningful (S) information is efficient (S) in the domain of efficiency (S).

In it's domain of efficiency, a meaningful information can participate to the determination of an action aimed at the system's constraint satisfaction. Outside of it's domain of efficiency, the meaningful information will still be meaningful but this meaning will not be usable for determining an action related to the satisfaction of the system's constraint. The information is meaningful but the meaning is not efficient.

In order to integrate this notion of efficiency to the various elements relative to meaningful information already introduced, we are going to consider a MGS attached to a transmitter that will transmit the meaningful information. We will name "transmitter system $\mathrm{T}$ " the global system containing the MGS and the transmitter. The transmitter system is submitted to a constraint T. We will suppose that the constraint $\mathrm{T}$ does not exist outside of the transmitter system. In the transmitter system, a meaningful information generated by the MGS is meaningful (T) and efficient (T).

When leaving the transmitter system, the meaningful information remains meaningful (T) but looses its efficiency. So the meaningful information transmitted is meaningful (T) and not efficient (T). Let's suppose that this meaningful ( $\mathrm{T}$ ) information reaches a receiver system $\mathrm{R}$ that is able to receive it. And let's also suppose that this receiver system contains a MGS having a constraint $\mathrm{R}$ that applies to the receiver system. The received information will be meaningful (T) and non efficient (T) in the receiver system. But if the received information has some connection with the constraint $\mathrm{R}$, then the receiver system will create a meaningful $(R)$ information that will be efficient $(R)$ in the receiver. Two meaningful informations then exist in the receiver system: a meaningful information that is meaningful (T) and non efficient (T), and another meaningful information that is meaningful (R) and efficient (R). In the receiver system, the meaningful (R) information can participate to the determination of an action in the direction of the receiver's constraint satisfaction. But the meaningful (T) information cannot participate in the receiver to the determination of an action relative to the constraint $(\mathrm{T})$. If the constraints $\mathrm{T}$ and $\mathrm{R}$ are identical, then the transmitter and the receiver belong to the same domain of efficiency, and the receiver can participate to the implementation of an action satisfying the constraint of the transmitter. 
Figure 3 summarizes these points:

Transmission of signal carrying information meaningful $(T)$ and non efficient $(T)$

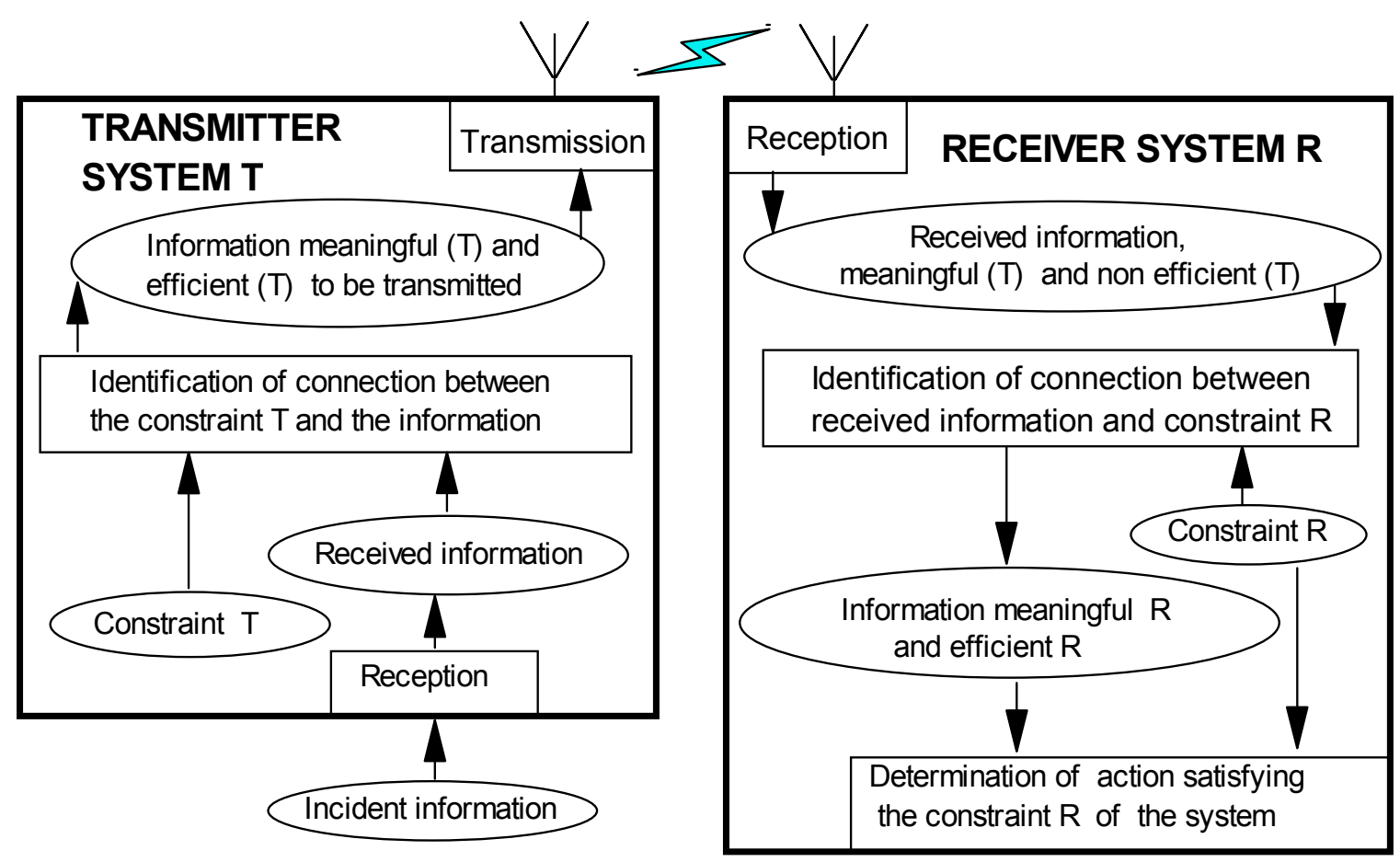

Figure 3. Transmission of a meaningful information.

This formalization of meaningful information transmission shows that a given meaningful information can generate different meanings in different systems receiving it. The actions implemented will depend upon the constraint of the systems receiving the information. Taking again the example of a male cicada calling female cicadas for reproduction, if we imagine that a "cicada eater" can hear the sound produced by the male cicada, he will find it and eat it. Cicadas and cicada eaters have different constraints to satisfy.

\section{Conclusion and Continuation}

We have introduced here an approach to the notion of "meaning" by defining a meaningful information, a meaning generator system (MGS) and the domain of efficiency of a meaning. The elements constituting the MGS are (Fig. 2):

- A system submitted to a constraint and able to receive an incident information.

- An information incident on the system.

- An information processing element, internal to the system and capable of identifying a connection between the received information and the constraint. 
A meaningful information (a "meaning") is defined as the connection existing between the received information and the constraint of the system. Properties of meaningful information and of MGS have been introduced.

This approach is to be continued by applying this systemic modelization to the different levels of complexity surrounding us (matter, life, mankind, machines). A key point will be the identification of the constraints of the systems at each level of complexity. More on this in http:/www.theorymeaning.fr.st.

\section{Notes and References}

1. C.S. Peirce (1839 - 1914) is considered as the source of the contemporary philosophical conception of semiotic - the science of signs -. Detailed information is available at Arisbe: http://members.door.net/arisbe/arisbe.htm (Arisbe, the Peirce Gateway, is a philosophical website providing coordinated access to the resources on the internet relevant to the life, work, and continuing interest in the American philosopher, scientist, and humanist Charles Sanders Peirce).

2. Gelepithis, P. A. M. Survey of Theories of Meaning. Cognitive Systems 1988, 2(2), 141-162. P. Gelepithis classifies the current theories of meaning in four categories (philosophical, linguistic, formal and biological). One conclusion is that these theories of meaning have recognized that meaning does depend on us humans.

3. A positioning of the notion of meaning in the history of Information Theory is available in J. Segal PhD Thesis 1998 (in French): Theorie de l'information: sciences, techniques et societe de la seconde guerre mondiale a l'aube du XXIe siècle. Text is in Chapt 11: La notion d'information dans l'emergence de l'unite du savoir. Internet version at: http://www.mpiwgberlin.mpg.de/staff/segal/thesis/thesehtm/chap11/ch11_.htm.

4. A. Sharov: Biosemiotics:functional-evolutionary approach to the analysis of the sense of information. In: Sebeok, T.A., J. Umiker-Sebeok (eds.): The Semiotic Web 1991: Biosemiotics. De Gruyter, Berlin-New York, 1992, 345-373.

(Internet version at: http://www.ento.vt.edu/\%7Esharov/biosem/txt/biosem.html.)

A. Sharov has analyzed the problem of the sense of information with the help of biosemiotics. Two aspects of the sense of an information are introduced: the meaning and the value. Meaning being the semantic characteristic of the sense, and value being the pragmatic characteristic. Biosemiotics has part of it's origins in the work of J. von Uexkull (1864 - 1944) who studied the problem of how living being subjectively perceive their environment and how this perception determines their behavior (with the key notion of "Umwelt"). Umwelt has been reactualized as a support for several studies on artificial life. Biosemiotics homepage at http://www.zbi.ee/ uexkull/biosem.htm.

(C) 2003 by MDPI (http://www.mdpi.org). Reproduction for noncommercial purposes permitted. 